J. Lake Sci. (湖泊科学), 2019, 31(6): 1489-1498

DOI 10. 18307/2019. 0625

(c) 2019 by Journal of Lake Sciences

\title{
人工湿地研究现状与展望”
}

\author{
成水平, 王月圆, 吴 娟 \\ (1:同济大学长江水环境教育部重点实验室,上海 200092) \\ (2: 同济大学环境生态工程研究所,上海 200092) \\ ( 3 : 上海生态景观水环境工程技术研究中心, 上海 200031)
}

\begin{abstract}
摘 要: 自 1950s 开始系统性研究以来, 人工湿地研究日趋深人, 也得到了广泛的应用. 本文基于文献报道, 对人工湿地 研究的历程特别是我国的研究进行了分析, 分为起步探索、迅猛发展和规范应用三个阶段. 梳理了人工湿地的研究现状, 主要集中在如何提高脱氮除磷效率、对新兴污染物的去除、人工湿地根区微生物结构与功能以及人工湿地模型四个方面. 核心还是提高人工湿地对污染物的去除效率并解释其机理. 未来需要长期监测数据支撑的理论实践结合的设计规程; 进 一步揭示人工湿地的生物过程;建立合理简化的模型，对系统进行准确预测.
\end{abstract}

关键词: 人工湿地;脱氮除磷; 新兴污染物; 微生物; 模型

\section{Advances and prospect in the studies on constructed wetlands}

CHENG Shuiping, WANG Yueyuan \& WU Juan

(1: Key Laboratory of Yangtze River Water Environment, Ministry of Education, Tongji University, Shanghai 200092, P.R. China)

(2: Institute of Eco-Environmental Engineering, Tongji University, Shanghai 200092, P.R.China)

(3: Shanghai Engineering Research Center of Landscape Water Environment, Shanghai 200031, P.R.China)

\begin{abstract}
Since the systematic research in the 1950s, the constructed wetland (CW) has attracted increasing attention and has been widely utilized. Based on the previous reports, we summarize the development of CW studies in China, and it is divided into three stages: initial exploration, rapid development and standardized application. In this review, measures of improving the efficiency of nitrogen, phosphorus and emerging pollutants removal, the microbial structure and function in the rhizosphere, and modeling of CW are summarized and discussed. In general, the key studies on CWs are to improve the removal efficiencies of pollutants and explain their mechanisms. In the future, a new design procedure combining theory and practice based on long monitoring data is needed, further exploration on the biological process in $\mathrm{CW}$ is necessary; a reasonable modeling would be established to accurately predict the $\mathrm{CW}$ process.
\end{abstract}

Keywords: Constructed wetland; nitrogen and phosphorus removal; emerging pollutants; microorganism; modeling

人工湿地 (Constructed Wetland, CW) 继承了湿地的水陆交汇处概念, 参与了人为因素, 由人工构筑而 成. 本文介绍的人工湿地是指在自然或半自然净化系统的基础上发展起来的水处理技术 ${ }^{[1]}$, 是一种人为地 将石、砂、土壤、煤渣等介质按一定比例构成的、且底部封闭，并有选择性植人水生植被的水处理生态系统， 水资源保护和持续利用是主要目的 ${ }^{[2]}$. 作为一种生态水处理方式, 人工湿地与传统水处理方式相比具有投 资低、运行费用少、耗能低, 且管理水平要求不高等优点, 近年来被广泛用来处理生活污水、工业废水、暴雨 径流、富营养化水体等, 并取得了良好效果; 但也存在占地面积较大、效率不高等不足，限制了其应用.

人工湿地通过基质、植物、微生物三者之间协同作用去除水体中污染物 ${ }^{[3-4]}$. 基质具有大的比表面积, 为 微生物的附着提供了良好的场所，同时基质可以通过吸附、离子交换等途径去除水体中的一部分污染物. 植

* 国家自然科学基金项目 (51578395,51778455) 资助. 2019-09-10 收稿; 2019-10-17 收修改稿. 成水平 ( 1969 ), 男,博士,教授;E-mail: shpcheng@ tongji.edu.cn. 
物对进人人工湿地中的污染物具有截留的作用; 并生长同化吸收的营养元素及其他污染物; 根系及根际分 泌物等构建根际微环境, 影响根际微生物群落结构与代谢. 微生物通过各种生理代谢途径将污染物从水体 中进行去除.

\section{1 发展历程}

德国较早系统地开展人工湿地的研究与应用. 1953 年 Seidel 研究发现芦苇能去除大量有机物和无机 物, 进一步的研究表明污水中的细菌在通过芦苇床时消失, 且芦苇及其他大型植物能从水中吸收重金属和 有机物等 ${ }^{[5-6]}$. 在此基础上 Kiehuth 提出了“根区法”, 1974 年, 完整的人工湿地在德国 Liebenburg-Othfresen 建成 ${ }^{[7]}$. 目前, 人工湿地技术已在全球广泛应用. 对人工湿地的研究热情也是与日俱增, 尤其是 2009 年以 后, 人工湿地英文发文数量每年迅速增加 (图 1). 截至 2018 年, 人工湿地累计发文量超过 10000 篇.

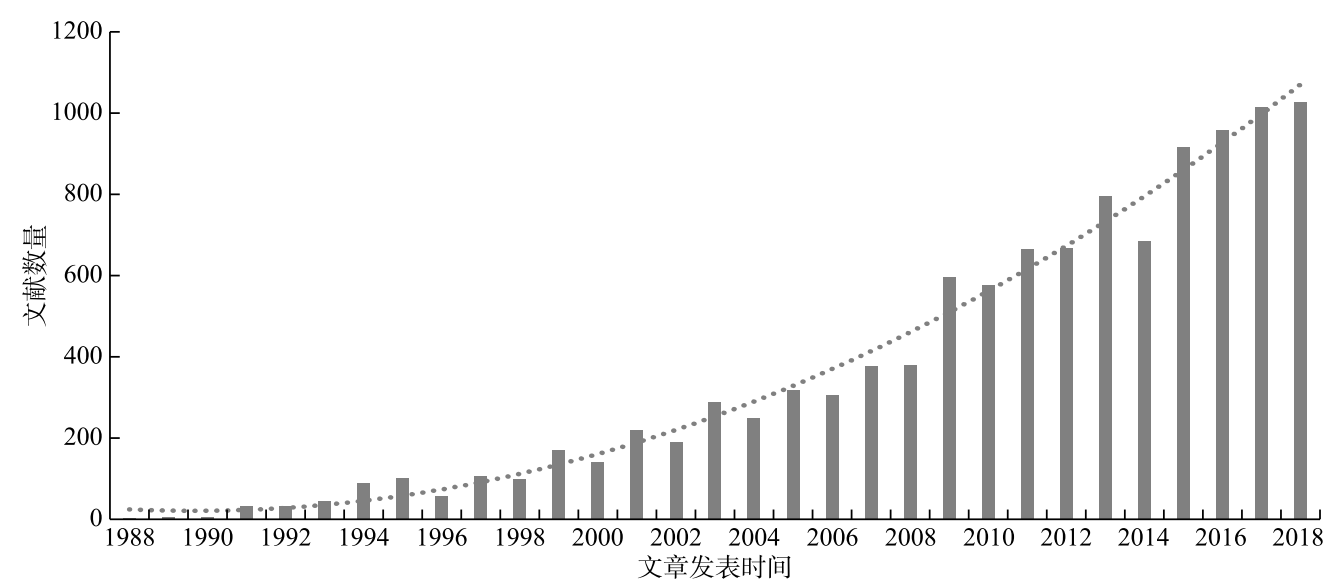

图 1 人工湿地英文文章数量

(来源: Web of Science, 搜索词: theme $=($ constructed wetland $*)$ or $($ artificial wetland $*)$ )

Fig.1 Papers published in English on constructed wetlands

相较于西方国家, 我国对人工湿地的研究起步较晚, 但是后来居上, 发表论文数量仅次于美国, 成为 第二大研究国家 (图 2). 我国人工湿地的研究与应用大致上可分为三个阶段: (1) 2000 年以前为起步探索阶 段. 随着生态治理技术的发展, 开始了稳定塘、土地处理系统、人工湿地的研究 ${ }^{[8-13]} .1990$ 年, 在深圳白泥坑 建设了生产性的人工湿地用于污水处理, 并以此为基地开展了湿地内部生物降解动力学、水力学等相关研 究 ${ }^{[10-11]}$, 北京昌平建成自由表面流人工湿地, 开展了一系列研究 ${ }^{[12]}$. (2) 2000- 2009 年为迅猛发展阶段. 随着水体污染控制与治理重大项目的实施, 我国人工湿地研究及应用得到了迅猛的发展, 论文数量迅速增 加, 研究范围涉及到重金属、藻类、澡毒素、农药和酞酸酯等污染物的去除 ${ }^{[1-18]}$, 人工湿地广泛应用于生活 污水、造纸废水、矿山废水、养殖废水、农业面源污染等污水的处理, 污水处理厂尾水的深度处理, 湖泊河流 等水体的生态修复, 小流域综合整治等. (3) 2009 年至今为规范应用阶段. 城乡与住房建设部和环境保护部 分别在 2009 年和 2010 年发布了人工湿地相关技术规范 ${ }^{[19-20]}$, 人工湿地在我国各类水处理中得到广泛的应 用, 同时也将水处理人工湿地与景观、生态环境保护与修复相结合, 既强调水处理效果, 也注重景观与生态 效应.

\section{2 主要研究内容及现状}

经过这几十年的发展, 人工湿地研究主要集中在如何提高脱氮除磷效率、对新兴污染物的去除、人工湿 地根区的微生物结构与功能、人工湿地模型等方面.

\section{1 人工湿地脱氮除磷效率提升}

人工湿地投资省、运行简, 但随着土地资源日益紧张, 人工湿地水力停留时间长, 占地面积大的缺点逐 


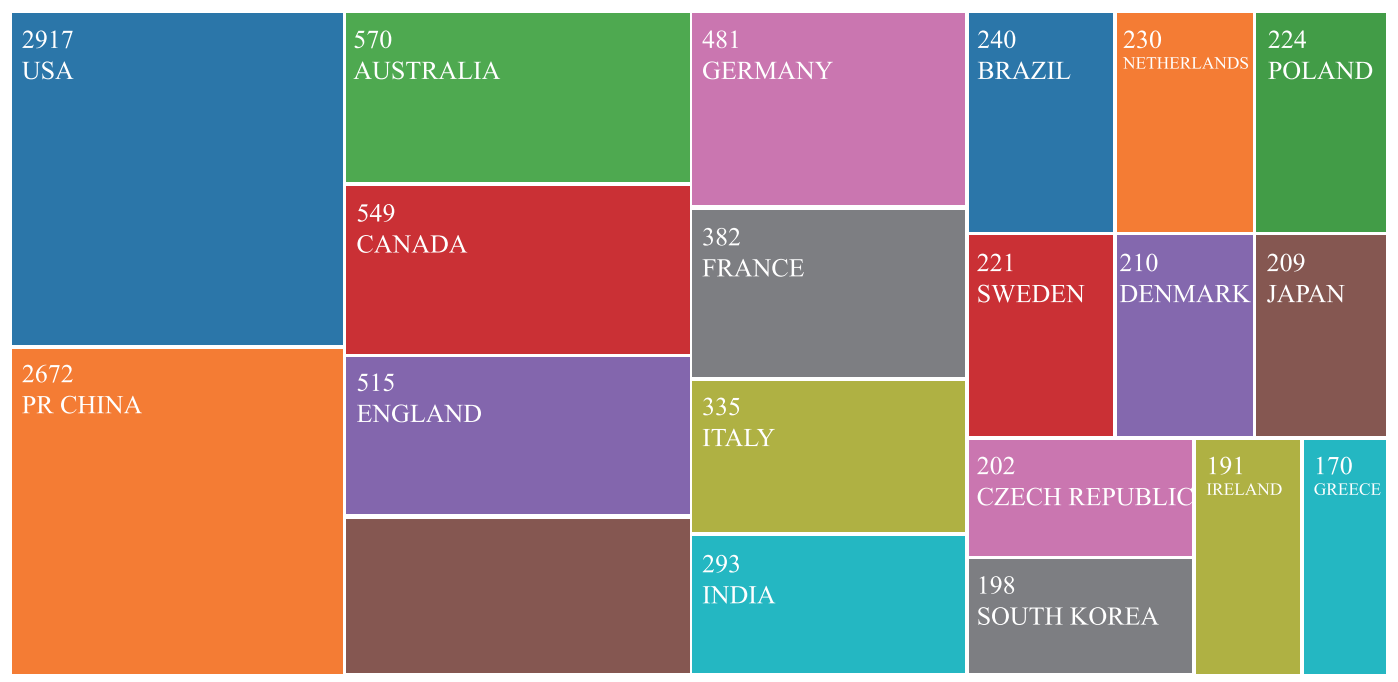

图 2 人工湿地英文文章全球分布

(来源: Web of Science, 搜索词: theme $=($ constructed wetland $*)$ or $($ artificial wetland $*))$

Fig. 2 Global distribution of English papers on constructed wetlands

渐不可忽视. 提高人工湿地脱氮除磷效率, 加大水力负荷, 减少占地面积就成为研究的热点问题. 主要在四 个不同方向来提升人工湿地脱氮除磷效率.

2.1.1 高效基质篎选与应用人工湿地选取的基质种类、粒径不同, 导致内部水力特性不同, 局部微环境存 在差异, 微生物群落结构各有特色, 同时, 不同的基质对污染物的截留吸附效能也不同, 从而影响人工湿 地的处理效率. 张翔凌等研究了不同组合的沸石、无烟煤、蛭石、高炉钢渣、生物陶粒等填料对垂直流人工湿 地水处理效果影响, 结果表明组合填料的种类、装填顺序和装填方式对净化效果产生了较大影响. 组合填料 对 COD 的平均去除率较单一填料都有所提高; 由上至下依次填充无烟煤、生物陶粒、沸石的组合填料具有较 好的脱氮功能; 由上至下依次填充无烟煤、蛭石、钢渣的组合填料具有较好的除磷功能 ${ }^{[21]}$. 不同粒径的沸 石、无烟煤、砾石人工湿地对总氮和总磷的去除率差异显著, 去除效率最高的分别是 $1 \sim 2 \mathrm{~mm}$ 沸石和 $2 \sim 4$ $\mathrm{mm}$ 无烟煤 ${ }^{[22]}$. 以给水厂脱水铝污泥泥饼作为潮汐流人工湿地的填料, 在处理养殖废水时, COD 、氨氮和总 磷的平均去除率分别达到 70\%、90\% 和 $90 \%$ 以上 ${ }^{[23]}$.

高效基质篮选的目的是提高人工湿地的污染物去除效能, 但要注意解决基质寿命问题. 基质通过吸附、 离子交换等方式去除水体中的污染物, 一旦吸附饱和, 人工湿地处理效率就会显著下降, 因此要进行基质 的更换, 且需要妥善处理. 再者, 基质应廉价易得. 在实际应用中, 基质投资占用了潜流人工湿地的大部分 建设费用, 基质选择应在效率和成本之间寻求一个合适的平衡点.

2.1.2人工湿地组合工艺 按照水流方式, 人工湿地可分为表面流人工湿地 (Surface flow constructed wetland, SFCW) 、水平潜流人工湿地 (Horizontal subsurface flow constructed wetland, HFCW) 和垂直流人工湿 地 (Vertical flow constructed wetland, VFCW) 等. 三种基本类型人工湿地各有优劣, 为提高人工湿地效率, 加 大负荷, 各种组合工艺应用而生. 应用最广泛的几种组合工艺有: 表面流与潜流人工湿地组合工艺, 水平潜 流与垂直潜流人工湿地组合工艺以及多级垂直流人工湿地组合工艺等.

表面流一潜流人工湿地应用于城镇分散污水和面源污染处理时, 出水水质满足《城镇污水处理厂污染 物排放标准》(GB 18918-2002) 一级 A 标准, 前端的潜流单元有利于去除污水中的 COD 、总磷和氨氮, 后 端的潜流单元有利于去除污水中的总氮 ${ }^{[24]}$. 而水平潜流一表面流人工湿地处理城镇污水处理厂尾水时, 在 5-10 月出水 $\mathrm{COD} \leqslant 20 \mathrm{mg} / \mathrm{L}$, 氨氮和总磷质量浓度分别小于 1.5 和 $0.3 \mathrm{mg} / \mathrm{L}^{[25-26]}$.

水平潜流与垂直潜流复合型人工湿地应用于地中海国家度假村的生活污水处理, 夏季高温期间对 
COD 、氮磷等具有良好的去除效果, 同时也能有效地降低出水中的致病菌浓度 ${ }^{[27]}$. 在爱沙尼亚, 寒冷气候下 水平潜流与垂直潜流复合型人工湿地处理生活污水, 出水满足排放标准要求 ${ }^{[28]}$.

多级垂直流人工湿地处理生活污水时，对 COD、总氮、总磷的脱除效率分别为 $90.1 \% 、 53.7 \%$ 和 $43.7 \%$, 并且通过分级进水, 当二级进水比例为 $20 \%$ 时, 该系统对总氮去除效率可提高至 $61.7 \%^{[29-30]}$. 水温对多级 垂直流湿地脱氮效率比有影响，总氮的去除率随温度升高呈上升趋势 ${ }^{[31]}$.

通过不同流态人工湿地工艺组合, 有利于提高人工湿地系统运行的稳定性, 对低温/高温均有一定的 适应性. 但是组合人工湿地的选取和组合方式大多依据经验, 其处理效率波动也较大, 如何保证人工湿地 组合系统的去除效果稳定持久也是一个难题. 已建成的组合人工湿地缺乏长期的现场跟踪监测数据.

2.1.3 与其他工艺的耦合 微生物在人工湿地去除污染物的过程中发挥重要作用, 为了进一步地强化微生 物作用, 强化人工湿地的脱氮除磷效率, 研究人员借鉴其他工艺发展成果, 将人工湿地与其他水处理工艺 相耦合.

将微生物燃料电池 (Microbial fuel cell, MFC) 加人到人工湿地中, 形成人工湿地一微生物燃料电池耦合 系统 (CW-MFC) 就是其中之一. 微生物燃料电池耦合人工湿地工艺的处理效果、产电效能等受到植物、运行 条件等各种因素的影响. 研究表明种植植物有利于 CW-MFC 系统阴极区域溶解氧浓度提升, 有利于 提高有 机物的降解 ${ }^{[32]}$. 在 CW-MFC 系统中, 进水 COD 较低时 $(50 \sim 250 \mathrm{mg} / \mathrm{L})$, 功率密度上升; 但当 COD 提高到 $250 \sim 1000 \mathrm{mg} / \mathrm{L}$ 时, 功率密度却呈下降趋势 ${ }^{[33]}$. 功率密度还受水力停留时间 (HRT) 的影响, 研究表明 HRT 在 6.5 13.1 h 时, MFC 的功率密度随 HRT 的增大而增大, 而当 HRT 在 13.1 50 h 时, MFC 的功率密度却 随 HRT 的增大而减小 ${ }^{[34]}$.

也有将人工湿地和电极生物膜反应器 (Biofilm electrode reactor, BER) 进行耦合. 生物膜电极反应器可 以在不需要外加碳源的条件下高效地去除低碳氮比废水中的硝酸盐. 系统对氮的去除效率受碳氮比、HRT 和 $\mathrm{pH}$ 等影响, 在 $\mathrm{C} / \mathrm{N}=0.75 \sim 1, \mathrm{HRT}=12 \mathrm{~h}, \mathrm{pH}=7.5$ 的条件下, 最终出水的最佳总氮和氨氮去除率分别 为 $63.0 \%$ 和 $98.1 \%{ }^{[35]}$. 人工湿地耦合生物膜反应器, 提高了氨氮和硝氮的去除效率, 从而降低了系统出水 总氮浓度.

有研究将人工湿地和高效藻池工艺相结合, 高效藻池水深较浅, 采用多廊道的形式, 利用藻类的光合 作用和微生物代谢处理污水, 同时回收生物质. Ding 等研究了高效藻池一水平潜流人工湿地系统对于模拟 废水的处理效果, 氨氮、硝氮和总氮去除效率均明显高于单一的人工湿地, 有效提高了系统的脱氮效率 ${ }^{\left[{ }^{[6]}\right.}$.

人工湿地和其他工艺耦合可以提高人工湿地的处理效率, 但是目前这些研究主要限于小试系统, 实验 条件严格控制, 实验周期也较短, 需要放大到中试或者小规模现场实验进行进一步考量. 同时, 耦合技术 的成本核算鲜有报道.

2.1.4 碳源补充 人工湿地应用于污水处理厂尾水的处理越来越多, 但污水处理厂尾水的一个典型特点就 是碳/氮比较低, 碳源不足影响到人工湿地脱氮效率, 因此人工湿地碳源补充也是一个重要的研究方向.

人工湿地碳源补充可分为三类, 一是可溶性碳源, 如甲醇、果糖和污泥等. 向人工湿地中补充甲醇使得 碳氮比达到 3:1, 总氮去除率为 $81 \% \sim 98 \%$, 显著高于未添加甲醇的系统 $(<10 \%)^{[37]}$. 二是固体碳源, 即不 溶性的合成类可生物降解有机物. Shen 等以玉米淀粉/聚己内酯 (PCL) 共混物作为人工湿地的补充碳源, 提高了系统的脱氮效率, 其中反硝化过程主要发生在 PCL 填充层中, 测序结果也显示反硝化细菌在该层为 优势菌 ${ }^{[38]}$. 采用聚-3-差基丁酸酯-共-3-差基戊酸酯 (PHBV) 和聚乙酸 (PGA) 的共混物作为碳源和生物膜载 体, 得到了与 Shen 等类似的结果 ${ }^{[39]}$. 三是植物性碳源, 如芦苇杆、稻壳等. 赵联芳等对比了芦苇杆、二球悬 铃木树叶和葡萄糖作为补充碳源对垂直流人工湿地脱氮效果的影响, 评估了不同碳源对 $\mathrm{N}_{2} \mathrm{O}$ 产生量的影 响, 分析了不同碳源湿地基质中微生物的优势菌群, 结果表明植物性碳源有助于提高系统脱氮效率, 但是 $\mathrm{N}_{2} \mathrm{O}$ 释放量要高于葡萄糖组, 三组实验中细菌均为基质中的优势群落 ${ }^{[40]}$.

可溶性碳源投加量不易控制, 且在人工湿地中很容易通过好氧降解, 不能长时间发挥作用. 固体性碳 源需提前预埋在人工湿地基质中, 效果稳定, 但不易补充, 且成本昂贵. 植物性碳源廉价易得, 可是效果不 稳定, 容易受到干扰, 同时会导致出水色度增加. 寻找廉价稳定, 方便补充, 可长期发挥作用的碳源, 并解 释其作用机理, 评估其生态效应, 将是一个重要任务. 


\section{2 去除新兴污染物}

随着化学合成技术日益提升, 环境中各类新兴污染物质层出不穷, 这些污染物往往具有较长的半衰 期, 可生化性能较差, 常规污水处理难以去除. 人工湿地微环境多样, 研究人工湿地对新兴污染物的去除 效果及规律也是一个热点方向.

表 1 列举了一些潜流人工湿地对双酚 $\mathrm{A}$ 和药品 及个人护理品 (Pharmaceuticals and personal care products, PPCPs) 等污染物的去除研究, 都表现出很 高的去除效率. 人工湿地对有机磷杀虫剂、菊酯类杀 虫剂、毒死蜱、二嗪农、西马津、草甘膦等农药的去除 效果分别为 $\approx 100 \% 、 95 \% \sim 99 \% 、 83 \% \sim 99 \% 、 68 \%$ $94 \% 、 20 \% \sim 60 \% 、 75 \% \sim 99 \%{ }^{[17,46-52]}$. 可见人工湿地对 去除新型污染物有很大的潜力. 多项研究表明, 人工 湿地对新型污染物的去除效率受到人工湿地的构 型、植物、水力停留时间、运行方式等多种因素的影 响 ${ }^{[53-56]}$. Zhang 等通过水培实验研究了双氯芬酸和咖 啡因在湿地植物水苾中的代谢, 发现双氯芬酸主要 通过光降解去除，而咖啡因则是被植物大量吸收， 且大量分布在茎叶中 ${ }^{[57-58]}$.

提高人工湿地对新兴污染物的去除效率是一个 重要研究趋势, 解释人工湿地去除机理也很重要, 是代谢分解还是被基质吸附对于生态环境的影响不 一. 如果是吸附, 则要防止长期运行之后基质二次污 染问题. 如果是代谢分解, 还要检测其降解产物, 解

表 1 人工湿地对部分新兴污染物的去除效率

Tab.1 Removal efficiency of emerging contaminants by constructed wetlands

\begin{tabular}{|c|c|c|c|}
\hline 污染物 & 人工湿地类型 & 处理效率 & 参考文献 \\
\hline \multirow{2}{*}{ 双酚 A } & 水平潜流 & $27.9 \% \sim 98.6 \%$ & [41-42] \\
\hline & 垂直流 & $42.9 \% \sim 100 \%$ & [43-44] \\
\hline 双酚 F & 垂直流 & $99.5 \%$ & {$[45]$} \\
\hline 双酚 S & 垂直流 & $99.9 \%$ & {$[45]$} \\
\hline \multirow{2}{*}{$\begin{array}{c}\text { 四溴双酚 A } \\
\text { 壬基酚 }\end{array}$} & 垂直流 & $99.9 \%$ & {$[45]$} \\
\hline & 水平潜流 & $20.4 \% \sim 90.0 \%$ & {$[41]$} \\
\hline \multirow{2}{*}{ 布洛芬 } & 垂直流 & $95.4 \% \sim 99.7 \%$ & {$[43]$} \\
\hline & 水平潜流 & $98.8 \%$ & {$[42]$} \\
\hline \multirow{2}{*}{ 双氯芬酸 } & 垂直流 & $54.3 \% \sim 70.25 \%$ & {$[43]$} \\
\hline & 水平潜流 & $100 \%$ & {$[42]$} \\
\hline \multirow{2}{*}{ 吐纳鹿香 } & 垂直流 & $61.1 \% \sim 83.3 \%$ & {$[43]$} \\
\hline & 水平潜流 & $97.8 \%$ & {$[42]$} \\
\hline 氧苯酮 & 垂直流 & $88.8 \% \sim 97.3 \%$ & {$[43]$} \\
\hline 三氯生 & 垂直流 & $72.7 \% \sim 88.6 \%$ & {$[43]$} \\
\hline 萘普生 & 水平潜流 & $99.1 \%$ & {$[42]$} \\
\hline
\end{tabular}
析降解途径, 部分新兴污染物的降解中间产物毒性甚至要高于母体, 要尽量减少有毒中间产物的形成.

\section{3 人工湿地根区微生物研究}

微生物在人工湿地去除污染物方面发挥着重要作用, 开展人工湿地 “黑箱” 中微生物研究是人工湿地 去除机理研究的重要一环. Nikolaos 等应用定量 PCR 对水平潜流人工湿地中的基因丰度进行测定, 根据基 因丰度判断人工湿地中氮去除的主要途径为硝化一反硝化 ${ }^{[59]}$. 熊家晴等通过 PCR-DGGE 技术对垂直流人工 湿地基质中各级微生物群落特性进行分析, 结果表明根际微生物群落多样性高于相同垂直深度的基质微生 物群落, 且出现了严格好氧菌, 证明植物根际具有泌氧功能 ${ }^{[60]}$. 雷旭等采用同样的技术对复合垂直流人工 湿地中不同植物根际微生物群落进行分析, 不同植物根际微生物不同, 且有季节差异 ${ }^{[61]}$.

随着高通量测序技术的成熟发展，应用高通量测序研究湿地中微生物群落结构的报道也与日俱增. Zhong 等采用 PCR-DGGE 和高通量测序比较研究了水平潜流人工湿地中微生物群落动态, 高通量测序能更 好地反映人工湿地中微生物群落的多样性 ${ }^{[62]}$. 高通量测序表明变形菌门人工湿地多于自然湿地, 而绿弯菌 门则是自然湿地多于人工湿地, 且自然湿地中的细菌群落呈现出更高多样性 ${ }^{[63]}$. 基质微生物多样性与植物 生物量密切相关, 研究表明植物根区微生物多样性明显增加, 放线菌丰度为 $20.9 \%$, 显著高于对照组 (无植 物) 的 $1.9 \%$, 并通过路径分析, 发现植物通过影响基质 $\mathrm{pH}$ 从而影响微生物的多样性 ${ }^{[64]}$.

通过高通量测序, 可以分析湿地中微生物的群落结构及多样性, 进一步梌释湿地中微生物的分布、污 染物对湿地微生物的影响、不同植物的根际微生物差异等, 有助于揭示污染物降解机理. 鉴于人工湿地系统 的复杂性, 微生境的多样性, 其中可能存在大量尚未解析的微生物, 功能不甚明确. 因此对人工湿地微生 物的研究除了对现有功能菌的研究, 也要注重未知菌属的作用. 大量存在某功能基因并不代表该菌群在人 工湿地系统中会发生作用, 需进行转录组和代谢产物的检测, 确认其是否发挥作用. 此外, 对人工湿地微 生物的研究大多局限于单个系统, 缺乏多个系统之间的对比, 难以发掘人工湿地系统中共有菌属和功能菌.

\section{4 人工湿地模型研究}

人工湿地作为一个黑箱, 机理研究可以使 “黑箱” 变“白箱”, 模型研究则是对黑箱的行为进行预测分 
析. 模型研究有助于工程设计的专业化, 也有助于对机理的进一步探究. 对于模型的研究, 大致可概括为以 下几类.

一是污染物去除的反应动力学. 例如采用一级动力学模型对水平潜流人工湿地和复合垂直流人工湿地 效率进行模拟比较, 模拟计算出水平潜流湿地 COD 、氨氮、总氮和总磷的面积速率常数分别为 $0.101 、 0.029$ 、 0.020 和 $0.121 \mathrm{~m} / \mathrm{d}$; 而复合垂直流湿地各污染物的面积速率常数分别为 $0.137 、 0.061 、 0.038$ 和 $0.197 \mathrm{~m} / \mathrm{d}$, 复合垂直流人工湿地效率显著高于水平潜流人工湿地 ${ }^{[65]}$. 也有采用 Monod 和多 Monod 动力学对水平潜流 人工湿地和垂直流人工湿地中氮和有机物的去除进行模拟, 分析进水碳氮比对模型参数的影响 ${ }^{[6]}$.

二是人工湿地的水力学计算模型. 通过示踪剂实验, 应用多流分散模型建模, 证明了水平潜流湿地中 存在多流系统, 这影响了湿地系统对氯苯的去除效率 ${ }^{[67]}$. Giraldi 等则利用纵向色散活塞流模型探讨了垂直 流人工湿地床层在不同饱和状态下的停留时间分布 ${ }^{[68]}$. 结合水力学和动力学过程, Pálfy 等开发了面向工 程师的 Orage 系统 ${ }^{[69]}$.

三是其他领域的模型或者活性污泥模型在人工湿地中的应用. 研究者应用 WASP 模型对表面流人工湿 地进行模拟, 对模型参数进行率定修正, 模拟值与实测值吻合度较高 ${ }^{[70-71]}$. 现在发展较为成熟的一个人工 湿地模型则是 Hydrus 模型. Hydrus 模型是基于活性污泥的 ASM 模型、结合人工湿地水力、生化特性开发的 一个模型, 包括 CW2D 和 CWM1 两个模块 ${ }^{[72]}$. CW2D 对处理生活污水的垂直流人工湿地进行模拟, 氨氮、 硝态氮和 COD 模拟值和实测值之间的平均绝对误差分别为 $27 \% 、 21 \%$ 和 $15 \%{ }^{[73]}$. CWM 1 对非稳态运行的水 平流人工湿地进行模拟, 模拟结果预测系统对 COD 的平均去除效率为 $68 \%$, 实测值为 $67 \%$, 两者十分

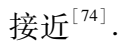

反应动力学和水力学计算模型较为简单, 对湿地内部各个过程进行简化, 参数少, 易于率定, 操作简 便, 可能更适用于工程设计. 基于人工湿地去污机理建立的模型更接近实际情况, 参数繁多, 系数选取受 系统影响较大, 但是对人工湿地内部的认识程度要高于前者. 这类模型的发展受人工湿地机理研究的影响 较大, 二者相辅相成. 目前, 在人工湿地模型研究方面, 精确度较高的模型实验规模小, 而针对实际工程应 用的模型则简化程度高, 这两者之间需要寻求一个平衡点, 或者说分道扬镀, 侧重不同预测方向. 总之, 人 工湿地模型发展是一个必然趋势.

\section{3 展望}

人工湿地作为一种生态处理技术，不仅改善水质，还具明显生态效益. 鉴于我国地表水污染现状，人 工湿地在污水处理厂尾水、城市黑臭水体、农村污水的治理及河道、湖泊的治理与修复保护等方面有广大的 应用前景. 如前文所述, 对于人工湿地的研究与应用仍有很多不明朗之处, 研究层面针对单一问题, 针对 人工湿地处理性能提升、各类污染物去除、机理研究和模型预测等方面做了大量工作, 而在实践中人工湿地 作为一个综合系统, 需要全面考量, 同时科研中缺乏实践数据反馈, 实验系统放大难以达到预期效果, 导 致人工湿地的处理效果在应用层面和研究层面出现差异, 并未发挥人工湿地的巨大潜力.

综上, 人工湿地以下工作还需要我们深人研究和实践:

1) 理论和实践数据相结合的设计规程. Kadlec 和 Wallace 撰写《Treatment Wetlands》一书, 详细介绍了 各类人工湿地的设计运行及管理 ${ }^{[75]}$. 但是这个设计手册中的参数大多基于国外人工湿地的监测结果, 而对 于我国而言, 还需要长期的实践数据来支撑. 我国城乡与住房建设部和环境保护部分别出台了人工湿地相 关技术规范 ${ }^{[19-20]}$. 工程设计依赖于现场的长期监测数据, 也依赖于对机理的进一步阐释, 同时也需要模型 的发展, 即要逐步从经验向有理有据的理论设计转变. 随着人工湿地应用的蓬勃发展, 越来越强调与景观 的结合, 通过植物配置来优化景观格局, 需要环境工程和风景园林专业交叉共同研究, 这也是人工湿地设 计应用要重点考虑的方向.

2) 人工湿地机理研究. 通过一些表观可控因子的调节已经不能满足提高人工湿地处理效率的要求, 人 工湿地去污机理越来越受到重视, 研究者需从更深一层的角度去解释去污机理, 从而提高人工湿地的处理 效率. 目前研究越来越集中于采用高通量测序, 分析湿地内部的微生物的一些特性, 如微生物多样性、群落 结构差异等. 人工湿地中植物的作用很为重视, 尤其是对植物根际的研究, 根际泌氧、根表鉄膜、根系分泌 
物等也有相关报道.

3) 人工湿地模型建立. 人工湿地的模型研究方兴未艾. 现有的研究大多为灰箱模型, 对于人工湿地内 部的过程作了太多的简化和假设, 从而导致了模型参数不具有广泛性. 而在实际人工湿地中, 条件复杂, 影响因子繁多, 导致模型预测值与真实值仍存在较大差异. 因此, 模型如何能提高预测精确度, 从而指导 设计, 辅助解释机理, 会成为模型发展的重要内容.

人工湿地经过几十年的发展, 技术日榛成熟, 但远未成为一个完善水处理系统. 人工湿地作为一个基 质、微生物和植物三者共同作用的复合系统, 其复杂程度远胜过单一生化系统, 研究难度也更大, 但随着研 究的深人进行, 人工湿地也将进一步发挥其巨大潜力, 在水处理和生态修复与保护方面发挥巨大的作用.

\section{4 参考文献}

[ 1 ] Fetter CW Jr, Sloey WE, Spangler FL. Potential replacement of septic tank drain fields by artificial marsh wastewater treatment systems. Ground Water, 1976, 14(6) : 396-401.

[ 2 ] Cheng SP. Advances in biological fundamental studies on artificial wetland wastewater treatment system. J Lake Sci, 1996, 8(3) : 268-273. DOI: 10.18307/1996.0312. [成水平. 人工湿地污水处理系统的生物学基础研究进展. 湖泊科学, $1996,8(3): 268-273$. ]

[ 3 ] Vymazal J, Krpfelová L. Removal of organics in constructed wetlands with horizontal sub-surface flow : A review of the field experience. Science of the Total Environment, 2009, 407(13) : 3911-3922.

[ 4 ] Cheng SP, Wu ZB, Kuang QJ. Macrophytes in artificial wetland. J Lake Sci, 2002, 14(2) : 179-184. DOI: 10.18307/ 2002.0213. [成水平, 吴振斌, 况琪军. 人工湿地植物研究. 湖泊科学, 2002, 14(2): 179-184.]

[ 5 ] Seidel K. Abgau von bacterium coli durch hohere wasser pflanzen. Naturwiss, 1964, 51 : 395.

[ 6 ] Seidel K. Reinigung von Gewassern durch hohere pflanzen. Naturwiss, 1966, 53: 289-297.

[ 7 ] Kickuth R. A low cost process for purification of municipal and industrial wastewater. Dertropenlandwirt, 1980, 83: 141-154.

[ 8 ] Zhu T, Xu ZC, Hu KP et al. Application research of constructed wetland sewage treatment system. Research of Environmental Sciences, 1991, 4(5): 17-22. [朱彤, 许振成, 胡康萍等. 人工湿地污水处理系统应用研究. 环境科学研究, $1991,4(5): 17-22$. ]

[ 9 ] Huang SD, Leng B. The technique of man-made wetland treatment system. Sichuan Environment, 1993, 12(2): 48-51. [黄时达, 冷冰. 污水的人工湿地系统处理技术. 四川环境, 1993, 12(2) : 48-51.]

[10] Chen YZ, Ye JL. Shenzhen Bainikeng and Yantian constructed wetland sewage treatment site. Electric Power Environmental Protection, 1996, (1): 47-51. [陈皿真, 叶纪良. 深圳白泥坑、雁田人工湿地污水处理场. 电力环境保护, 1996, (1) : 47-51.]

[11] Wang JX. Study on hydraulic calculation of artificial wetland in Bainikeng. Guangdong Water Resources and Hydropower, 1997, (6) : 50-52. [王久贤. 白泥坑人工湿地水力学计算研究. 广东水利水电, 1997, (6) : 50-52.]

[12] Chinese Environmental Protection Agency, Division of Science and Technology and Standarded. Technical guideline on the land treatment for municipal wastewater. Beijing: China Environmental Science Press, 1997: 166. [国家环境保护局科 技标准司. 城市污水土地处理技术指南. 北京: 中国环境科学出版社, 1997: 166.]

[13] Cheng SP, Kuang QJ, Xia YC. Studies on artificial wetland with cattail (Typha angustifolia) and rush (Juncus effusus) (I): The performance of purification wastewater. J Lake Sci, 1997, 9(4) : 351-358. DOI: 10.18307/1997.0410. [成水 平, 况琪军, 夏宜琤. 香蒲、灯心草人工湿地的研究 I. 净化污水的效果. 湖泊科学, 1997, 9(4) : 351-358.]

[14] Cheng SP, Grosse W, Karrenbrock F et al. Efficiency of constructed wetlands in decontamination of water polluted by heavy metals. Ecological Engineering, 2002, 18(3) : 317-325.

[15] Kuang QJ, Wu ZB, Xia YC. Studies on the removal efficiency of algae by constructed wetland. Acta Hydrobiologica Sinica, 2000, 24 (6) : 655-658. [况琪军, 吴振斌, 夏宜琤. 人工湿地生态系统的除藻研究. 水生生物学报, 2000, 24(6): 655-658.]

[16] Wu ZB, Chen HR, Lei LM et al. Study of the effect of constructed wetland on the removal of microcystins. Resources and Environment in the Yangtze Basin, 2000, (2) : 242-247. [吴振斌, 陈辉蓉, 雷腊梅等. 人工湿地系统去除藻毒素研 究. 长江流域资源与环境, 2000, (2) : 242-247.] 
[17] Cheng SP, Vidakoviz-Cifrek Ž, Grosse W et al. Xenobiotics removal from polluted water by multifunctional constructed wetland. Chemosphere, 2002, 48(4) : 415-418.

[18] Liang W, Wu ZB, Zhou QH et al. Study on the microorganism and enzyme mechanism of constructing wetland to remove phthalate. Resources and Environment in the Yangtze Basin, 2003, (3): 254-258. [梁威, 吴振斌, 周巧红等. 构建湿地 去除唒酸酯的基质微生物和酶机制研究. 长江流域资源与环境, 2003，(3)：254-258.]

[19] Institute of Standard and Rating, Ministry of Housing and Urban-Rural Development of the People's Republic of China. Technical guidelines for wastewater treatment of constructed wetlands [RISNTG006-2009]. Beijing: China Architecture and Building Press, 2009: 188. [中华人民共和国住房和城乡住建部标准定额研究所. 人工湿地污水处理技术导则 [RISNTG006-2009]. 北京: 中国建筑工业出版社, 2009: 188.]

[20] Ministry of Ecology and Environment of the People's Republic of China. Technical specification of constructed wetlands for wastewater treatment engineering. [HJ 2005-2010]. Beijing: China Environmental Science Press, 2010: 16. [中华人民 共和国环境保护部. 人工湿地污水处理工程技术规范 [ HJ 2005-2010]. 北京: 中国环境科学出版社出版, 2010: 16.]

[21] Zhang XL, Wu JM, Wang R et al. Purification capacity of different combined fillers in vertical flow constructed wetlands. China Water and Wastewater, 2018, 25(19) : 1-4. [张翔凌, 武俊梅, 王荣等. 垂直流人工湿地系统中不同组合填料 净化能力研究. 中国给水排水, 2008, 25(19): 1-4.]

[22] Zhao LL, Shao XX, Wu M et al. Effects of different substrates and particle size on purification of wastewater. Environmental Science, 2018, (9) : 1-8. [赵林丽, 邵学新, 吴明等. 人工湿地不同基质和粒径对污水净化效果的比较. 环境科 学, 2018, (9): 1-8.]

[23] Zhao YQ, Zhao XH, Babatunde AO. Use of dewatered alum sludge as main substrate in treatment reed bed receiving agricultural wastewater: Long-term trial. Bioresource Technology, 2009, 100(2) : 644-648.

[24] Chen Y, Chen YH, Song H et al. Treatment effectiveness of sewage drain into Xingyue Reservoir by surface flow constructed wetlands - subsurface flow wetlands - subsurface flow wetlands. Wetland Science, 2018, 16(1) : 59-66. [ 陈荣, 陈永 华, 宋胡等. 表面流一潜流一潜流串联人工湿地对排人星月水库污水的处理效果. 湿地科学, 2018, 16 (1): 59-66. ]

[25] Xiong JQ, Duan R, Zheng YC et al. Research on the purification differences of polluted river water treated by different forms of hybrid constructed wetlands. Industrial Water Treatment, 2017, 37(10): 27-30. [熊家晴, 段然, 郑于聪等. 不 同形式复合人工湿地对污染河水的净化差异研究. 工业水处理, 2017, 37(10): 27-30.]

[26] Hu J, Xu GY, Hu X et al. Tail water advanced treatment by hybrid constructed wetlands in a small town wastewater treatment plant. Technology of Water Treatment, 2018, (11)：120-122, 132. [胡洁, 许光远, 胡香等. 组合式人工湿地深 度处理小城镇污水处理厂尾水. 水处理技术, 2018, (11): 120-122, 132.]

[27] Masi F, Martinuzzi N. Constructed wetlands for the Mediterranean countries: hybrid systems for water reuse and sustainable sanitation. Desalination, 2007, 215(1/2/3): 44-55.

[28] Mander Ü, Antti T, Mauring T et al. Performance dynamics of a LWA-filled hybrid constructed wetland in Estonia. Ecohydrology \& Hydrobiology, 2007, 7(3/4) : 297-302.

[29] Tang MX, Wu J, Dai YR et al. Treatment of domestic wastewater by hybrid vertical constructed wetland. Chinese Journal of Environmental Engineering, 2016, 10(3) : 1017-1022. [ 唐孟煊, 吴娟, 代嫣然等. 组合式垂直流人工湿地工艺及其 污水处理效果. 环境工程学报, 2016, 10(3): 1017-1022.]

[30] Shen LY, Wu J, Zhong F et al. Effect of step feeding on the performance of multi-stage vertical flow constructed wetland for municipal wastewater treatment. J Lake Sci, 2017, 29(5) : 1084-1090. DOI:10.18307/2017.0506. [沈林亚, 吴娟, 钟非等. 分级进水对阶梯垂直流人工湿地污水处理效果的影响. 湖泊科学, 2017, 29(5): 1084-1090.]

[31] Feng L, Lu BH, Wang YC et al. Effect of water temperature of multi-stage vertical-flow constructed wetland on pollutants removal from domestic wastewater. Technology of Water Treatment, 2015, 41(9): 87-91. [冯蕾, 路博华, 王运超等. 多 级垂直流人工湿地水温对去除生活污水污染物的影响. 水处理技术, 2015, 41(9): 87-91.]

[32] Fang Z, Song H, Cang N et al. Performance of microbial fuel cell coupled constructed wetland system for decolorization of azo dye and bioelectricity generation. Bioresource Technology, 2013, 144(6) : 165-171.

[33] Liu S, Song H, Wei S et al. Bio-cathode materials evaluation and configuration optimization for power output of vertical subsurface flow constructed wetland-microbial fuel cell systems. Bioresource Technology, 2014, 166(8) : 575-583. 
[34] Sharma Y, Li B. Optimizing energy harvest in wastewater treatment by combining anaerobic hydrogen producing biofermentor (HPB) and microbial fuel cell (MFC). International Journal of Hydrogen Energy, 2010, 35( 8) : 3789-3797.

[35] He Y, Wang Y, Song X. High-effective denitrification of low $\mathrm{C} / \mathrm{N}$ wastewater by combined constructed wetland and biofilm electrode reactor (CW-BER). Bioresource Technology, 2016, 203: 245.

[36] Ding Y, Wang W, Liu X et al. Intensified nitrogen removal of constructed wetland by novel integration of high rate algal pond biotechnology. Bioresource Technology, 2016, 219: 757-761.

[37] Huett DO, Morris SG, Smith G et al. Nitrogen and phosphorus removal from plant nursery runoff in vegetated and unvegetated subsurface flow wetlands. Water Research, 2005, 39(14) : 3259-3272.

[38 ] Shen Z, Zhou Y, Liu J et al. Enhanced removal of nitrate using starch/PCL blends as solid carbon source in a constructed wetland. Bioresource Technology, 2015, 175: 239-244.

[39] Yang Z, Yang L, Wei C et al. Enhanced nitrogen removal using solid carbon source in constructed wetland with limited aeration. Bioresource Technology, 2017, 248(Part B) : 98-103.

[40] Zhao LF, He L, Mei CH et al. Denitrifying and denitrifying effect and $\mathrm{N}_{2} \mathrm{O}$ release from an external plant carbon source constructed wetland system. Journal of Safety and Environment, 2018, 18(1):276-281. [赵联芳, 贺丽, 梅才华等. 外 置植物碳源型人工湿地系统反硝化脱氮效果及 $\mathrm{N}_{2} \mathrm{O}$ 释放. 安全与环境学报, 2018, 18(1): 276-281.]

[41] Toro-Vélez AF, Maderaparra CA, PeñaVarón MR et al. BPA and NP removal from municipal wastewater by tropical horizontal subsurface constructed wetlands. Science of the Total Environment, 2016, 542 ( Pt A) : 93-101.

[42] Avila C, Pedescoll A, Víctor M et al. Capacity of a horizontal subsurface flow constructed wetland system for the removal of emerging pollutants: An injection experiment. Chemosphere, 2010, 81(9) : 1137-1142.

[43] Avila C, Nivala J, Olsson L et al. Emerging organic contaminants in vertical subsurface flow constructed wetlands: Influence of media size, loading frequency and use of active aeration. Science of the Total Environment, 2014, 494-495: 211-217.

[44] Riry W, Kazuhiro M, Tadashi T. Effect of activated carbon on removal of four phenolic endocrine-disrupting compounds, bisphenol A, bisphenol F, bisphenol S and 4-tert-butylphenol in constructed wetlands. Chemosphere, 2018, 210:717-725.

[45] Park N, Vanderford BJ, Snyder SA. Effective controls of micropollutants included in wastewater effluent using constructed wetlands under anoxic condition. Ecological Engineering, 2009, 35(3) : 418-423.

[46] Budd R, O'Green A, Goh KS et al. Efficacy of constructed wetlands in pesticide removal from tailwaters in the Central Valley, California. Environmental Science \& Technology, 2009, 43(8) : 2925-2930.

[47] Moore MT, Schulz R, Copper CM et al. Mitigation of chlorpyrifos runoff using constructed wetlands. Chemosphere, 2002, 46(6): 827-835.

[48] Moore MT, Copper CM, Smith S et al. Diazinon mitigation in constructed wetlands: Influence of vegetation. Water, Air and Soil Pollution, 2007, 184(1-4) : 313-321.

[49] Agudel RM, Jaramillo ML, Peñuela G. Comparison of the removal of chlorpyrifos and dissolved organic carbon in horizontal sub-surface and surface flow wetlands. Science of the Total Environment, 2012, 431(1) : 271-277.

[ 50 ] Maillard E, Sylvain P, Etienne F et al. Removal of pesticide mixtures in a stormwater wetland collecting runoff from a vineyard catchment. Science of the Total Environment, 2011, 409(11): 2317-2324.

[51] Declan P, Peter D, Jochen M et al. Quantification of herbicide removal in a constructed wetland using passive samplers and composite water quality monitoring. Chemosphere, 2010, 81(3) : 394-399.

[52] Matamoros V, Puigagut J, Garcia J et al. Behavior of selected priority organic pollutants in horizontal sub-surface flow constructed wetlands: A preliminary screening. Chemosphere, 2007, 69(9) : 1374-1380.

[53] Jing RY, Yang Y, Dai YN et al. Removal behavior of ibuprofen and diclofenac in different constructed wetlands. Environmental Science, 2016, 37(7): 2577-2585. [景瑞瑛, 杨扬, 戴玉女等. 布洛芬和双氯芬酸在不同构型人工湿地中的 去除行为研究. 环境科学, 2016, 37(7): 2577-2585.]

[54] Xu QL, Cui LH. Removal efficiency of dimethyl phthalate by vertical flow constructed wetlands. Wetland Science, 2017, 15(2) : 298-301. [许巧玲, 崔理华. 垂直流人工湿地对邻苯二甲酸二甲酯的去除效果研究. 湿地科学, 2017, 15 (2) : 298-301.]

[55] Liao P, Zhan ZY, Dai J et al. Adsorption of tetracycline and chloramphenicol in aqueous solutions by bamboo charcoal: A batch and fixed-bed column study. Chemical Engineering Journal, 2013, 228: 496-505. 
[56] Zhang DQ, Gersberg RM, Zhu J et al. Batch versus continuous feeding strategies for pharmaceutical removal by subsurface flow constructed wetland. Environ Pollut, 2012, 167: 124-131.

[57] Zhang DQ, Hua T, Gersberg RM et al. Fate of diclofenac in wetland mesocosms planted with Scirpus validus. Ecological Engineering, 2012, 49(12) : 59-64.

[58] Zhang DQ, Hua T, Gersberg RM et al. Fate of caffeine in mesocosms wetland planted with Scirpus validus. Chemosphere, $2013,90(4)$ : 1568-1572.

[59] Paranychianakis NV, Tsiknia M, Kalogerakis N. Pathways regulating the removal of nitrogen in planted and unplanted subsurface flow constructed wetlands. Water Research, 2016, 102: 321-329.

[60] Xiong JQ, Li SS, Ge Y et al. Characterization of microbial communities in vertical flow constructed wetland for highly polluted river water treatment. Chinese Journal of Environmental Engineering, 2017, 11(3): 1959-1965. [ 熊家晴, 李珊珊, 葛媛等.处理高污染河水垂直流人工湿地微生物群落特性. 环境工程学报, 2017, 11(3) : 1959-1965.]

[61] Lei X, Li B, Li X et al. Rhizosphere microbial communities of three plants in vertical-flow constructed wetland. Chinese Journal of Ecology, 2015, 34(5): 1373-1381. [雷旭, 李冰, 李晓等. 复合垂直流人工湿地系统中不同植物根际微 生物群落结构. 生态学杂志, 2015, 34(5): 1373-1381.]

[62] Zhong F, Wu J, Dai Y et al. Bacterial community analysis by PCR-DGGE and 454-pyrosequencing of horizontal subsurface flow constructed wetlands with front-aeration. Applied Microbiology and Biotechnology, 2015, 99(3) : 1499-1512.

[63] Ansola G, Arroyo P, Sáenz de Miera LE. Characterization of the soil bacterial community structure and composition of natural and constructed wetlands. Science of the Total Environment, 2014, 473-474(3) : 63-71.

[64] Chen Y, Wen Y, Tang Z et al. Effects of plant biomass on bacterial community structure in constructed wetlands used for tertiary wastewater treatment. Ecological Engineering, 2015, 84: 38-45.

[65] Fan YH. The simulation of contaminants removal in the constructed wetlands. Railway Energy Saving \& Environmental Protection \& Occupational Safety and Health , 2018，8(1): 20-25. [范英宏. 人工湿地污染物去除动力学模拟研究. 铁路 节能环保与安全卫生, 2018, 8(1): 20-25.]

[66] Saeed T, Sun G. Kinetic modelling of nitrogen and organics removal in vertical and horizontal flow wetlands. Water Research, 2018, 45(10): 3137-3152.

[67] Birkigt J, Stumpp C, Małoszewski P et al. Evaluation of the hydrological flow paths in a gravel bed filter modeling a horizontal subsurface flow wetland by using a multi-tracer experiment. Science of the Total Environment, 2018, 621: 265.

[68] Giraldi D, Vitturi MD, Zaramella M et al. Hydrodynamics of vertical subsurface flow constructed wetlands: Tracer tests with rhodamine WT and numerical modelling. Ecological Engineering, 2009, 35(2): 265-273.

[69] Pálfy TG, Gourdon R, Meyer D et al. Model-based optimization of constructed wetlands treating combined sewer overflow. Ecological Engineering, 2017, 101: 261-267.

[70] Chen DK, Zhu WB, Wang HX et al. Optimization of WASP water quality model and its application to free water surface constructed wetland systems. Industrial Water Treatment, 2018, 38(2): 70-74. [陈德坤, 朱文博, 王洪秀等. WASP 水 质模型在表流人工湿地中的优化与应用. 工业水处理, 2018, 38(2): 70-74.]

[71] Wang HX, Yuan ZD, Wu ZH et al. COD removal efficiency and application of WASP model to Wuhe constructed wetland. China Water \& Wastewater, 2014, 30(11) : 69-72. [王洪秀, 袁佐栋, 武周虎等. 武河人工湿地工程去除 COD 效果 及 WASP 模拟. 中国给水排水, 2014, 30(11): 69-72.]

[72] Meyer D, Chazarenc F. Modelling constructed wetlands: Scopes and aims-A comparative review. Ecological Engineering, 2015, 80: 205-213.

[73] Morvannou A, Choubert JM, Vanclooster M et al. Modeling nitrogen removal in a vertical flow constructed wetland treating directly domestic wastewater. Ecological Engineering, 2014, 70(5) : 379-386.

[74] Rizzo A, Langergraber G, Galvão A et al. Modelling the response of laboratory horizontal flow constructed wetlands to unsteady organic loads with HYDRUS-CWM1. Ecological Engineering, 2014, 68(7) : 209-213.

[75] Kadlec R, Wallace S. Treatment wetlands. Boca Raton: CRC Press, 2009: 1016. 